\title{
Genome Sequence of Idiomarina sp. Strain A28L, Isolated from Pangong Lake, India ${ }^{\nabla}$
}

\author{
Hemant Kumar Gupta, ${ }^{1}$ \# Ajit Singh, ${ }^{1}$ \# and Rakesh Sharma ${ }^{1,2 *}$ \\ Microbial Biotechnology and Genomics, Institute of Genomics and Integrative Biology, Council of Scientific and \\ Industrial Research (CSIR), Mall Road, Delhi-110007, India, ${ }^{1}$ and National Chemical Laboratory, Council of \\ Scientific and Industrial Research, Dr. Homi Bhabha Road, Pune-411008, India ${ }^{2}$
}

Received 24 June 2011/Accepted 29 June 2011

\begin{abstract}
Idiomarina sp. strain A28L was isolated from the alkaline brackish water of a high-altitude lake, Pangong Lake. Here, we present the draft genome of Idiomarina sp. strain A28L, which contains 2,591,567 bp with a G+C content of 45.5 mol\% and contains 2,299 protein-coding genes and 56 structural RNAs.
\end{abstract}

The genus Idiomarina belongs to the family Idiomarinaceae of the class Gammaproteobacteria. The genus contained 19 species at the time of writing (12). All of these bacteria were isolated from saline habitats with a wide range of salinities, such as coastal and oceanic waters, coastal sediments, submarine hydrothermal fluids, solar salt-making works, and inland hypersaline wetlands (5). Strain A28L was isolated by culture on alkaline bacillus agar ( $\mathrm{pH} 9.5$ ) containing $1.5 \% \mathrm{NaCl}$ from the alkaline brackish water of Pangong Lake, which is situated at an altitude of $4350 \mathrm{~m}$ in Ladakh, India. This strain is a Gram-negative, aerobic, flagellar bacterium. Phylogenetic analysis of the rrs (16S rRNA) gene sequence revealed that strain A28L belongs to the genus Idiomarina. It showed the highest $r r$ gene sequence identity, $95.2 \%$, with the type strain of Idiomarina maris (12).

The genome of Idiomarina sp. strain A28L was sequenced by a whole-genome shotgun strategy using 454 GS FLX (Roche) at the Institute of Genomics and Integrative Biology, Delhi, India. A total of 282,412 sequence reads were generated by FLX Titanium sequencing with a genome coverage of 38-fold. The sequence was assembled into 31 contigs by Newbler software, version 2.3 (Roche). The 28 large contigs ( $>500$ bp to 394,143 bp) were used for gene prediction and annotation by the IMG ER system (7), which uses Prodigal (4) for gene prediction. The IMG ER automated pipeline uses a homology search at the nonredundant proteins database (NR) of GenBank, pfam (2), and the TIGRFam (9), InterProScan (11), KEGG (6), and COG (10) databases for annotation (8). Annotated coding sequences from the IMG ER server were used for a round of manual curation by BLAST, and the noncoding RNA and RNA with miscellaneous features were analyzed by Rfam, before submission of the annotated draft genome to GenBank. The draft genome sequence was also uploaded to the RAST (1) server.

The Idiomarina sp. strain A28L unclosed draft genome was $2,591,567 \mathrm{bp}$ in length, with a $\mathrm{G}+\mathrm{C}$ content of $45.5 \mathrm{~mol} \%$. The genome, which has a coding density of $90 \%$, contained 2,299

\footnotetext{
* Corresponding author. Mailing address: Institute of Genomics and Integrative Biology, Council of Scientific and Industrial Research (CSIR), Mall Road, Delhi-110007, India. Phone: 91-11-27666156. Fax: 91-11-27667471. E-mail: rsharma@igib.res.in.

\# These authors contributed equally to this work.

${ }^{\nabla}$ Published ahead of print on 8 July 2011.
}

protein coding genes, 49 tRNA genes, and 4 rRNA genes. Comparison with genome sequences available at RAST showed that I. loihiensis L2TR (score, 517), I. baltica OS145 (score, 430) followed by Pseudoalteromonas tunicata (score, 321) were the closest neighbors of strain A28L. The strain A28L genome contained genes for glycolysis/gluconeogenesis, the tricarboxylic acid cycle, a high-affinity phosphate transporter and regulatory genes for sensing inorganic phosphate, and genes for flagellum assembly and function, while the genes for sugar transporters (phosphotransferase system, ABC-type sugar transporter, and sugar-phosphate permease), the Entner-Doudoroff pathway, inorganic sulfur assimilation, and siderophore biosynthesis were absent, which was similar to the reported $I$. loihiensis L2TR genome (3). In contrast to $I$. loihiensis L2TR, genes for thiamine biosynthesis, ammonia assimilation, and denitrification were absent in strain A28L. Detailed comparative analyses of Idiomarina sp. strain A28L with I. loihiensis L2TR and I. baltica OS145 should reveal their metabolic differences and the genetic basis of adaptation to their unique habitats.

Nucleotide sequence accession numbers. This whole-genome shotgun project has been deposited at DDBJ/EMBL/ GenBank under the accession number AFPO00000000. The version described in this paper is the first version, AFPO01000000. The 28 large contigs have been deposited under accession numbers AFPO01000001 to AFPO01000028.

We thank Rajesh S. Gokhale, Director, I.G.I.B., for encouragement and support. We thank Konstantinos Mavrommatis, Joint Genome Institute, for providing data files from the IMG ER server.

H.K.G. acknowledges ICMR for a junior research fellowship. The work was supported by funding from the Institute of Genomics and Integrative Biology and NCL-IGIB-JRI project NWP013.

\section{REFERENCES}

1. Aziz, R., et al. 2008. The RAST server: Rapid Annotations using Subsystems Technology. BMC Genomics 9:75.

2. Finn, R. D., et al. 2008. The Pfam protein families database. Nucleic Acids Res. 36:D281-D288.

3. Hou, S., et al. 2004. Genome sequence of the deep-sea $\gamma$-proteobacterium Idiomarina loihiensis reveals amino acid fermentation as a source of carbon and energy. Proc. Natl. Acad. Sci. U. S. A. 101:18036-18041.

4. Hyatt, D., et al. 2010. Prodigal: prokaryotic gene recognition and translation initiation site identification. BMC Bioinformatics 11:119.

5. Jean, W. D., et al. 2009. Pseudoidiomarina marina sp. nov. and Pseudoidiomarina tainanensis sp. nov. and reclassification of Idiomarina homiensis and Idiomarina salinarum as Pseudoidiomarina homiensis comb. nov. and 
Pseudoidiomarina salinarum comb. nov., respectively. Int. J. Syst. Evol. Microbiol. 59:53-59.

6. Kanehisa, M., S. Goto, M. Furumichi, M. Tanabe, and M. Hirakawa. 2010 KEGG for representation and analysis of molecular networks involving diseases and drugs. Nucleic Acids Res. 38:D355-D360.

7. Markowitz, V. M., et al. 2009. IMG ER: a system for microbial genome annotation expert review and curation. Bioinformatics 25:2271-2278.

8. Mavromatis, K., et al. 2009. The DOE-JGI standard operating procedure for the annotations of microbial genomes. Stand. Genomic Sci. 1:63-67.

9. Selengut, J. D., et al. 2006. TIGRFAMs and Genome Properties: tools for the assignment of molecular function and biological process in prokaryotic genomes Nucleic Acids Res. 35:D260-D264.

10. Tatusov, R. L., M. Y. Galperin, D. A. Natle, and E. V. Koonin. 2000. The COG database: a tool for genome-scale analysis of protein functions and evolution. Nucleic Acids Res. 28:33-36.

11. Zdobnov, E. M., and R. Apweiler. 2001. InterProScan — an integration platform for the signature-recognition methods in InterPro. Bioinformatics 17:847-848.

12. Zhang, Y.-J., et al. 2011. Idiomarina maris sp. nov., a marine bacterium isolated from sediment of the South China Sea. Int. J. Syst. Evol. Microbiol. doi:10.1099/ijs.0.027896-0. 